\title{
Hyperbolische 5-Rechtecke
}

H. ZEITLER

Zur Erinnerung an den 200. Jahrestag der Geburt des weltberühmten ungarischen Geometers János Bolyai (1802-1860)

Abstract. The main topic of this paper is the investigation of 5-pentagons whose interior angles are all right angles within the hyperbolic geometry (so-called 5-rectangles). Some knowledge of elementary hyperbolic geometry is required.

At first the existence of such a polygon is shown by construction within the Kleinmodel. Then two formulas due to D. M. Y. Sommerville [3] are proved. This means to juggle with trigonometric formulas of hyperbolic geometry.

In the last years a big number of papers concerning hyperbolic geometry was published. This proves that the interest in this nice discipline is growing again.

Key words and phrases: hyperbolic geometry, Klein-model, right-angled pentagons (Hyperbolische Geometrie, Klein-Modell, rechtwinklige 5-Ecke).

ZDM Subject Classification: G90-4.

1. 5-Rechtecke-gibt es das überhaupt?

$n$-Ecke der hyperbolischen Geometrie mit $n$ rechten Innenwinkeln heißen $n$ Rechtecke.

\subsection{Notwendige Bedingung}

$n>4$ ist notwendige Bedingung für die Existenz von $n$-Rechtecken.

BEWEIS. Die Winkelsummre im $n$-Eck ist in der hyperbolischen Geometrie (kurz H-Geometrie) stets kleiner $(n-2) \cdot \pi$. Weil aber bei $n$-Rechtecken für die Winkelsumme $n \cdot \frac{\pi}{2}$ gilt, so folgt $n \cdot \frac{\pi}{2}<(n-2) \cdot \pi$, also $n>4$. 


\subsection{Flächeninhalt des 5-Rechtecks}

Der Flächeninhalt $F$ des 5-Rechtecks beträgt $\frac{1}{2} \pi$.

BeweIs. Die Fläche eines $H$-Dreiecks mit den Winkeln $\alpha, \beta, \gamma$ beträgt $k^{2}(\pi-$ $\alpha-\beta-\gamma)$. Dabei ist $k$ eine Normierungskonstante. Wir legen fest $k=1$.

Jedes 5-Rechteck läßt sich in drei H-Dreiecke zerlegen. Also ist seine Fläche (unabhängig von der Art der Zerlegung) gleich der Summe der drei Dreiecksflächen (Additivität der Inhalts-maßzahlen). Dies ergibt $F=F_{1}+F_{2}+F_{3}=$ $3 \pi-S$. Dabei ist $S$ die Summe aller 9 Dreieckswinkel. Wegen $S=5 \cdot \frac{\pi}{2}$ folgt also weiter $F=3 \pi-\frac{5}{2} \pi=\frac{1}{2} \pi$.

\subsection{Existenznachweis durch Konstruktion}

Es gibt hyperbolische 5-Rechtecke.

Daß die notwendige Bedingung aus 1.1 im Falle $n=5$ auch hinreichend ist, beweisen wir durch Konstruktion im Klein-Modell. Dazu ist die Kenntnis gewisser Grundkonstruktionen unbedingt erforderlich: H-Lot fällen, H-Senkrechte errichten, Spiegelung an einer H-Geraden vornehmen, einen H-Winkel halbieren, ... Ohne solche Zutaten bleibt unser Beweis unverständlich. Wir verweisen auf einschlägige Literatur.

Die Konstrukion in verschiedenen Schritten.

\section{Schritt (Figur 1)}

Eine H-Gerade $p_{1}$ mit zugehörigem Pol $P_{1}$, sowie ein $\mathrm{H}-\mathrm{Punkt} A_{1} \in p_{1}$ seien beliebig gegeben. Dann ist die H-Gerade auf $P_{1} A_{1}$ orthogonal zu $p_{1}$. Nun wird der rechte Winkel bei $A_{1}$ in der üblichen Weise hyperbolisch halbiert. Dies liefert die Gerade $g$.

2. Schritt (Figur 2)

Wir wählen einen Punkt $P_{2} \in g$ so, daß $P_{1} P_{2}$ eine Sekante des Grenzkreises im Klein-Modell ist. Weiter sei dann $p_{2}$ die zu $P_{2}$ gehörende Polare. Dann ist die H-Gerade auf $P_{1} P_{2}$ senkrecht zu $p_{1}$, aber auch zu $p_{2}$. Mit $g \cap p_{2}=\{A\}$ erhalten wir das H-Viereck $\left(A_{1} A_{2} A_{3} A\right)$ mit drei rechten Winkeln. Der vierte Winkel hat die Größe $\frac{1}{2} \cdot \frac{1}{2} \pi$. 


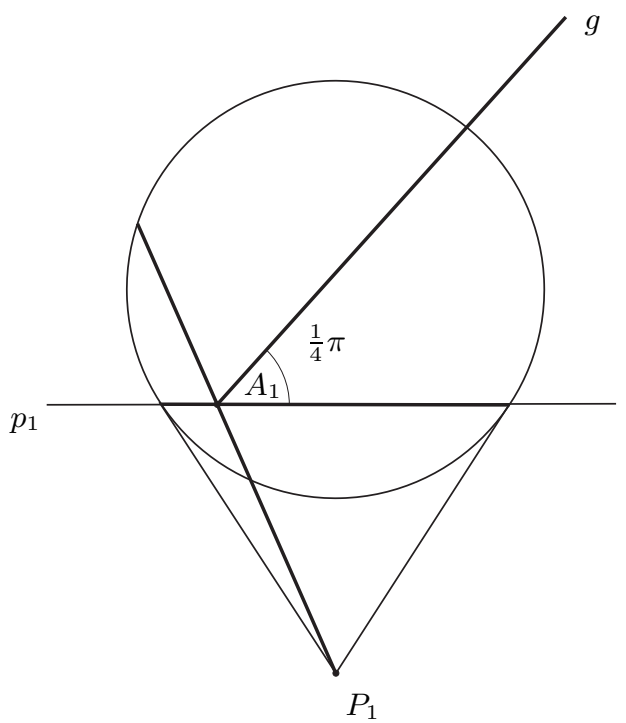

Figur 1. H-Senkrechte errichten und H-Winkel halbieren

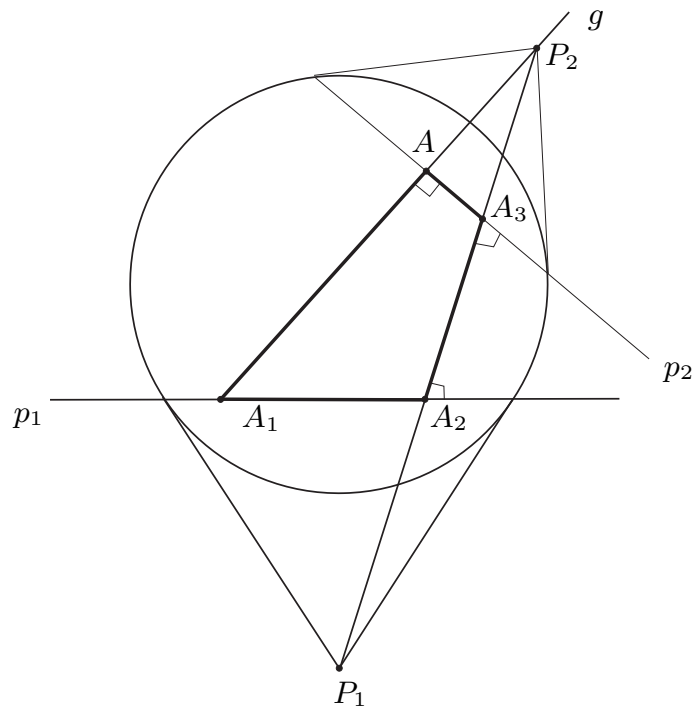

Figur 2. Ein H-Viereck mit drei rechten Winkeln 


\section{Schritt (Figur 3)}

H-Spiegelung des H-Vierecks an der H-Geraden auf $g$ liefert das 5-Rechteck $\left(A_{1} A_{2} A_{3} A_{4} A_{5}\right)$. Dabei sind $A_{4}$ bzw. $A_{5}$ die H-Spiegelungspunkte von $A_{3}$ und $A_{2}$. Die H-Punkte $A_{1}$ und $A$ bleiben fix.

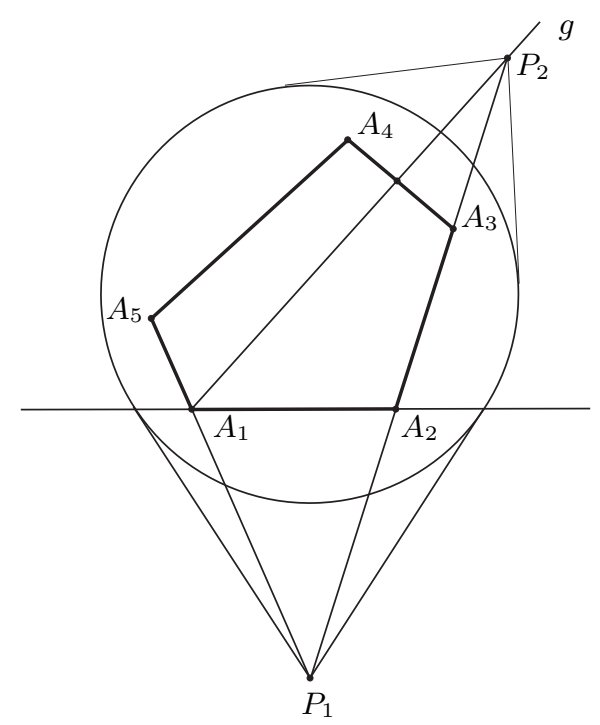

Figur 3. Ein 5-Rechteck

Damit ist die Existenz eines 5-Rechtecks nachgewiesen. Nach dem angegebenen Rezept kann sich jeder sein eigenes 5-Rechteck zusammenbasteln.

\subsection{Das regulär 5-Rechteck}

Figur 4 zeigt einen besonders hübschen Sonderfall. Nämlich ein 5-Rechteck mit 5 gleichlangen Seiten. Wir sprechen vom regulären 5-Rechteck.

\subsubsection{Wie wurde daß Bildchen konstruiert?}

In einen Kreis mit Mittelpunkt $M$ wird ein reguläres, überkreuztes 5-Eck $\left(P_{1} P_{2} P_{3} P_{4} P_{5}\right)$ einbeschrieben (Pentagramm). Zur Auffindung des Grenzkreises im Klein-Modell zeichnen wir den Thaleskreis über $M P_{1}$. Geschnitten mit $P_{2} P_{5}$ liefert das die Punkte $X$ und $Y$. Dann ist $M X$ der Radius des gesuchten Kreises 


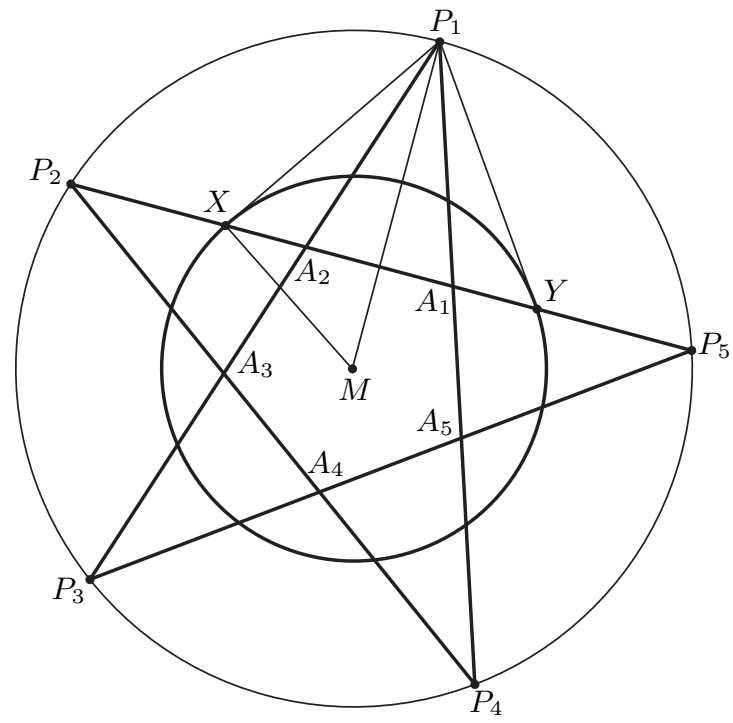

Figur 4. Ein reguläres 5-Rechteck

und $P_{1}$ der Pol zu $P_{2} P_{5}$. Die H-Geraden auf $P_{1} P_{3}$ und auf $P_{1} P_{4}$ stehen auf $X Y$ hyperbolisch senkrecht. Aus Symmetriegründen bilden die Schnittpunkte der Seiten unseres Startpolygons im Klein-Modell das reguläre 5-Rechteck $\left(A_{1} A_{2} A_{3} A_{4} A_{5}\right)$.

\subsubsection{Seitenlänge im regulären 5-Rechteck}

Für die hyperbolische Länge a einer Seite des regulären 5-Rechtecks gilt ch $a=$ $\frac{1}{2}(\sqrt{5}+1)$.

BEWEIS. In H-Dreiecken mit den Winkeln $\alpha, \beta, \gamma$ gilt der Winkelkosinussatz: $\cos \alpha=-\cos \beta \cos \gamma+\sin \beta \sin \gamma \operatorname{ch} a$. Wir wenden ihn an auf das H-Dreieck $\left(M A_{1} A_{2}\right)$ mit $\alpha=\frac{2}{5} \pi, \beta=\gamma=\frac{1}{4} \pi$ und erhalten $\operatorname{ch} a=\frac{\cos \frac{2}{5} \pi+\cos ^{2} \frac{1}{4} \pi}{\sin ^{2} \frac{1}{4} \pi}$.

Mit $\cos \frac{2}{5} \pi=\frac{1}{4}(\sqrt{5}-1)$ und $\cos ^{2} \frac{1}{4} \pi=\sin ^{2} \frac{1}{4} \pi=\frac{1}{2}$ folgt daraus $\operatorname{ch} a=$ $\frac{1}{2}(\sqrt{5}+1)$. Man nennt $\frac{1}{2}(\sqrt{5}+1)$ auch die goldene Zahl.

Bemerkungen zu Abschnitt 1. Bei der Konstruktion in 1.3 wurden zwei H-kongruente Spezial-Vierecke an einander gelegt. Jedes dieser Vierecke hat neben drei rechten Winkeln noch einen spitzen Winkel $\varepsilon$. Solche H-Vierecke nennt man Spitzecke oder Lambert Vierecke (nach Johann Heinrich Lambert, 1728-1777). In unserem Fall hatten wir $\varepsilon=\frac{1}{4} \pi$. 
Durch Zusammenfügen nicht kongruenter Lambert-Vierecke lassen sich folgende Aussagen bezüglich der Symmetrieachsen unsrer 5-Rechtecke gewinnen.

(a) Es gibt 5-Rechtecke ohne eine Symmetrieachse.

(b) Es gibt 5-Rechtecke mit genau einer Symmetrieachse.

(c) Wenn ein 5-Rechteck zwei Symmetrieachsen besitzt, dann sogar 5 und wir haben das reguläre 5-Eck aus 1.4.

Beweisen Sie die drei Aussagen im Detail!

\section{Formeln von D. M. Y. Sommerville [3]}

\subsection{Die Formeln}

Im 5-Rechteck gilt

ch (Seite)

= Produkt der sh-Werte der beiden nicht anliegenden Seiten

= Produkt der cth-Werte der beiden anliegenden Seiten.

Wir schreiben das mit den Bezeichnungen der Figur 5 explizit an.

$$
\begin{gathered}
\text { 1. Klasse } 2 . \text { Klasse } \\
\operatorname{ch} a=\operatorname{sh} c \operatorname{sh} d=\operatorname{cth} b \operatorname{cth} e \\
\operatorname{ch} b=\operatorname{sh} d \operatorname{sh} e=\operatorname{cth} c \operatorname{cth} a \\
\operatorname{ch} c=\operatorname{sh} e \operatorname{sh} a=\operatorname{cth} d \operatorname{cth} b \\
\operatorname{ch} d=\operatorname{sh} a \operatorname{sh} b=\operatorname{cth} e \operatorname{cth} c \\
\operatorname{ch} e=\operatorname{sh} b \operatorname{sh} c=\operatorname{cth} a \operatorname{cth} d .
\end{gathered}
$$

Es geht einfach von einer Zeile zur nächsten zyklisch weiter.

D. M. Y. Sommerville nennt diesen Satz und bezeichnet ihn als „Beispiel“. Den Beweis überläßt er dem Leser. Wir wollen hier einen Beweis in allen Details vorführen. 


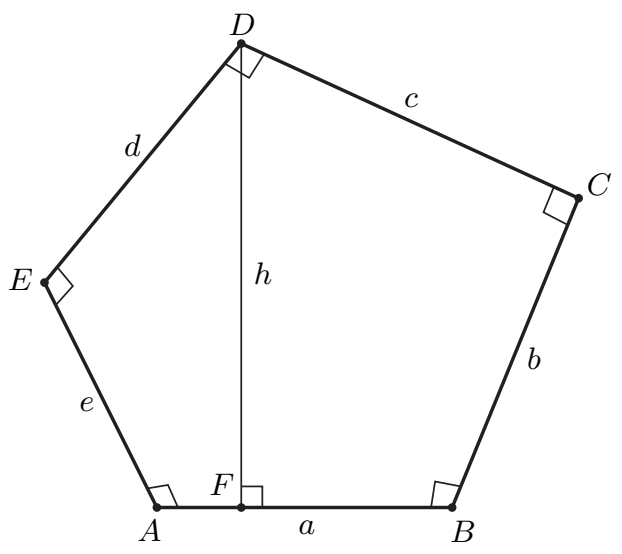

Figur 5. Ein hyperbolisches 5-Rechteck

\subsection{Die erste Klasse von Formeln}

2.2.1. Lambert-Vierecke.

Figur 6 zeigt ein H-Viereck mit drei rechten Winkeln und einem spitzen, also nach Definition ein Lambert-Viereck.

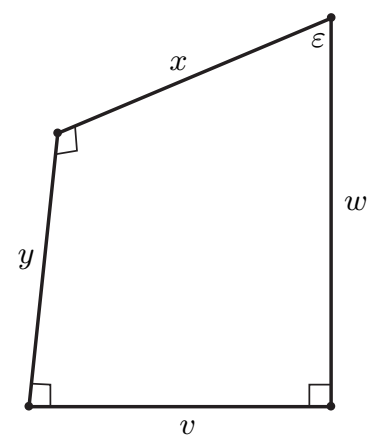

Figur 6. Ein Lambert-Viereck

In der Literatur finden sich Formeln, die einen Zusammenhang von Seitenlängen und dem spitzen Winkel $\varepsilon$ herstellen.

$$
\begin{aligned}
& \operatorname{sh} w=\operatorname{ch} x \operatorname{sh} y, \\
& \operatorname{sh} x=\operatorname{ch} w \operatorname{sh} v, \quad \operatorname{th} x=\operatorname{ch} y \operatorname{th} v, \quad \text { th } w=\operatorname{ch} v \operatorname{th} y
\end{aligned}
$$




$$
\cos \varepsilon=\operatorname{th} w \text { th } x
$$

Wir werden in dieser Arbeit nur die erste dieser Formeln verwenden.

\subsubsection{Zerlegung eines 5-Rechtecks}

Fällt man von der Ecke $D$ in Figur 5 das H-Lot auf die gegenüberliegende Seite, so entstehen zwei Lambert-Vierecke $(F D E A)$ und $(F B C D)$. Nach 2.2.1 gilt dann $\operatorname{sh} h=\operatorname{ch} d \operatorname{sh} e$ und weiter $\operatorname{sh} h=\operatorname{ch} c \operatorname{sh} b$. Dies aber bedeutet $\operatorname{ch} c \operatorname{sh} b=$ $\operatorname{ch} d \operatorname{sh} e$.

Weil die fünf Innenwinkel im 5-Rechteck alle recht sind, liegen die Fußpunkte der Lote von jeder Ecke auf die zugehörige Gegenseite stets im Innern dieser Seite. Deshalb lassen sich durch zyklische Vertauschung weitere Formeln gewinnen.

$$
\begin{array}{rlrl}
\text { I } & \operatorname{ch} d \operatorname{sh} e & =\operatorname{ch} c \operatorname{sh} b \\
\text { II } \quad \operatorname{ch} e \operatorname{sh} a & =\operatorname{ch} d \operatorname{sh} c \\
\text { III } \quad \operatorname{ch} a \operatorname{sh} b & =\operatorname{ch} e \operatorname{sh} d \\
\text { IV } \quad \operatorname{ch} b \operatorname{sh} c & =\operatorname{ch} a \operatorname{sh} e \\
\text { V } \quad \operatorname{ch} c \operatorname{sh} d & =\operatorname{ch} b \operatorname{sh} a .
\end{array}
$$

Für unsere Behauptung bezüglich der Lage der Fußpunkte skizzieren wir an Hand der Figur 5 einen Beweis.

Nehmen wir zunächst an, der Fußpunkt $F$ des Lotes von $D$ auf $A B$ falle mit $B$ zusammen. Dann liegen die Punkte $B=F, C$ und $D$ auf einer Geraden und es gilt $\varangle(D C B)=\pi$, im Widerspruch $\mathrm{zu} \varangle(D C B)=\frac{1}{2} \pi$.

Nun liege $F$ außerhalb der H-Strecke $A B$ - etwa rechts von $B$. Dann ist $\varangle(D C B)$ zwar recht, aber dieser Winkel (Umlaufsinn) wurde zu einem Außenwinkel. Dies steht im Widerspruch zur Definition des 5-Rechtecks mit fünf rechten Innenwinkeln.

Für die weiteren Rechnungen ist es vorteilhaft, die Formeln (2) zu quadrieren.

\subsubsection{Elimination von $a$}

Unser Ziel soll es jetzt sein, die Gleichung $\operatorname{ch} b=\operatorname{sh} d$ she zu beweisen. Zu diesem Zweck eliminieren wir jetzt $a$. Aus Gleichung IV in (2) folgt $\operatorname{ch}^{2} a=$ $\frac{\operatorname{ch}^{2} b \operatorname{sh}^{2} c}{\operatorname{sh}^{2} e}$ und weiter $\operatorname{sh}^{2} a=\operatorname{ch}^{2} a-1=\frac{\operatorname{ch}^{2} b \operatorname{sh}^{2} c-\operatorname{sh}^{2} e}{\operatorname{sh}^{2} e}$. Wir setzen das in die 
Formeln (2) ein und erhalten vier Gleichungen für $b, c, d, e$.

$$
\begin{array}{ll}
\mathrm{I}^{*} & \operatorname{ch}^{2} d \operatorname{sh}^{2} e=\operatorname{ch}^{2} c \operatorname{sh}^{2} b \\
\mathrm{II}^{*} & \operatorname{ch}^{2} e \operatorname{ch}^{2} b \operatorname{sh}^{2} c-\operatorname{ch}^{2} e \operatorname{sh}^{2} e=\operatorname{ch}^{2} d \operatorname{sh}^{2} c \operatorname{sh}^{2} e \\
\mathrm{III}^{*} & \operatorname{ch}^{2} b \operatorname{sh}^{2} c \operatorname{sh}^{2} b=\operatorname{ch}^{2} e \operatorname{sh}^{2} e \operatorname{sh}^{2} d \\
\mathrm{~V}^{*} & \operatorname{ch}^{2} c \operatorname{sh}^{2} d \operatorname{sh}^{2} e=\operatorname{ch}^{4} b \operatorname{sh}^{2} c-\operatorname{ch}^{2} b \operatorname{sh}^{2} e .
\end{array}
$$

\subsubsection{Elimination von $c$}

Zur Gewinnung von $\operatorname{ch} b=\operatorname{sh} d \operatorname{sh} e$ muß auch noch $c$ eliminiert werden.

Dies geschieht dadurch, daß wir in (3) statt $\operatorname{sh}^{2} c$ schreiben $\operatorname{ch}^{2} c-1$. Dann werden die Gleichungen III* und II* nach $\operatorname{ch}^{2} c$ aufgelöst und gleichgesetzt.

$$
\begin{aligned}
& \mathrm{II}^{*} \quad \operatorname{ch}^{2} c=1+\frac{\mathrm{ch}^{2} e \operatorname{sh}^{2} e}{\operatorname{ch}^{2} e \operatorname{ch}^{2} b-\operatorname{ch}^{2} d \operatorname{sh}^{2} e} \\
& \mathrm{III}^{*} \quad \operatorname{ch}^{2} c=1+\frac{\operatorname{ch}^{2} e \operatorname{sh}^{2} e \operatorname{sh}^{2} d}{\operatorname{ch}^{2} b \operatorname{sh}^{2} b} \\
& \mathrm{II}^{*}=\mathrm{III} \\
& \quad 1+\frac{\operatorname{ch}^{2} e \operatorname{sh}^{2} e}{\operatorname{ch}^{2} e \operatorname{ch}^{2} b-\operatorname{ch}^{2} d \operatorname{sh}^{2} e}=1+\frac{\operatorname{ch}^{2} e \operatorname{sh}^{2} e \operatorname{sh}^{2} d}{\operatorname{ch}^{2} b \operatorname{sh}^{2} b} \\
& \Longrightarrow \operatorname{ch}^{2} b \operatorname{sh}^{2} b-\operatorname{sh}^{2} d \operatorname{ch}^{2} e \operatorname{ch}^{2} b+\operatorname{sh}^{2} d \operatorname{ch}^{2} d \operatorname{sh}^{2} e=0 .
\end{aligned}
$$

Wir erwarten $\operatorname{ch} b=\operatorname{sh} d \operatorname{sh} e$ und schreiben deshalb

$$
\operatorname{ch}^{2} d=1+\operatorname{sh}^{2} d, \quad \operatorname{ch}^{2} e=1+\operatorname{sh}^{2} e, \quad \operatorname{sh}^{2} b=\operatorname{ch}^{2} b-1 .
$$

So erhalten wir

$$
\begin{gathered}
\operatorname{ch}^{2} b\left(\operatorname{ch}^{2} b-1\right)-\operatorname{sh}^{2} d\left(1+\operatorname{sh}^{2} e\right) \operatorname{ch}^{2} b+\operatorname{sh}^{2} d\left(1+\operatorname{sh}^{2} d\right) \operatorname{sh}^{2} e=0 \\
\Longrightarrow\left(\operatorname{sh}^{2} d \operatorname{sh}^{2} e-\operatorname{ch}^{2} b\right)\left(\operatorname{ch}^{2} b-1-\operatorname{sh}^{2} d\right)=0 \\
\Longrightarrow\left(\operatorname{sh}^{2} d \operatorname{sh}^{2} e-\operatorname{ch}^{2} b\right)\left(\operatorname{ch}^{2} b-\operatorname{ch}^{2} d\right)=0
\end{gathered}
$$

Im Falle $b \neq d$ erhalten wir also $\operatorname{ch}^{2} b=\operatorname{sh}^{2} d \operatorname{sh}^{2} e$ und weil alle ch- und sh-Werte positiv sind $\operatorname{sogar} \operatorname{ch} b=\operatorname{sh} d \operatorname{sh} e$. 


\subsubsection{Kritische Fälle}

Die Untersuchung des Falles $b=d$ erfordert eine grünliche Fallunterscheidung. Das läßt sich leider nicht vermeiden.

(a) 5 Seiten paarweise verschieden

Dann ist alles klar. Die noch fehlenden Formeln der ersten Klasse ergeben sich durch zyklische Vertauschung.

(b) 3 Seiten gleich lang

Da gibt es zwei in Figur 7 skizzierte Möglichkeiten.

$$
a=b=c=x .
$$

Mit IV aus (2) folgt $\operatorname{ch} x \operatorname{sh} x=\operatorname{ch} x$ sh $e$, also $e=x$.

$$
a=b=d=y .
$$

Mit $\mathrm{V}$ aus (2) gilt $\operatorname{ch} c \operatorname{sh} y=\operatorname{ch} y \operatorname{sh} y$, also $y=c$. Sind drei Seiten gleich, dann auch vier.

(c) 4 Seiten gleich lang

$$
a=b=c=d=x
$$

Mit I aus (2) folgt $\operatorname{ch} x \operatorname{sh} e=\operatorname{ch} x \operatorname{sh} x$, also $x=e$. Sind 4 Seiten gleich, dann auch alle 5. Wir haben es mit dem in 1.4 abgehandelten Fall des regulären 5-Rechtecks zu tun.

(d) 2 Seiten gleich lang

Das ist jetzt genau unser kritischer Fall $b=d=x$. Mit III aus (2) folgt $\operatorname{ch} a \operatorname{sh} x=$ $\operatorname{ch} e \operatorname{sh} x$, also $a=e$. Damit haben wir ein achsensymmetrisches 5-Rechteck (Figur 7).

Es gilt $b=d$ und $a=e$, aber auch $b \neq a, e$ und $d \neq a, e$. Denn sonst hätten wir ja 3 oder 4 gleichlange Seiten und damit ein reguläres 5-Rechteck. Dieser Fall ist aber bereits abgehandelt. Sei nun $d \neq a$. Dann folgt in völliger Analogie zu Abschnitt 2.2 die Gleichung $\operatorname{ch} d=\operatorname{sh} a \operatorname{sh} b$. Mit $d=b, a=e$ weiter $\operatorname{ch} b=\operatorname{sh} e \operatorname{sh} d$.

Damit ist die Richtigkeit der Gleichung $\operatorname{ch} b=\operatorname{sh} d \operatorname{sh} e$ auch für den Fall $b=d$ nachgewiesen.

Wir können also zusammenfassend sagen, daß die 5 Sommerville-Formeln der ersten Klasse in jedem 5-Rechteck gelten. 
Drei gleiche Seiten:

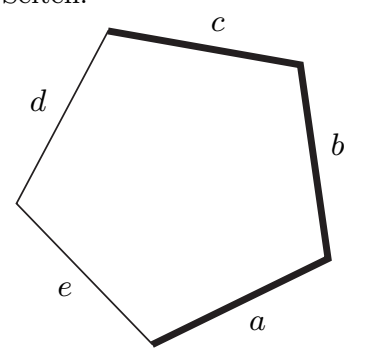

$a=b=c=x$

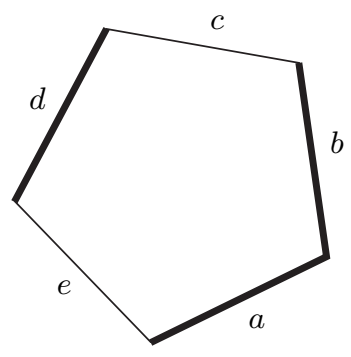

$a=b=d=y$

Zwei gleiche Seiten:

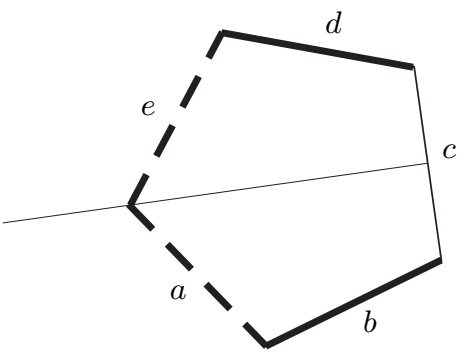

$b=d \quad a=e$

Figur 7. Sonderfälle

2.3. Die zweite Klasse von Formeln

Jetzt wenden wir uns der zweiten Klasse von Sommerville-Formeln zu und setzen dabei die Gültigkeit der Formeln aus der ersten Klasse voraus.

Unser bisheriges Vorgehen: In den Formeln (2) hatten wir in 2.2.3 zunächst $a$ und in 2.2.4 dann auch noch $c$ eliminiert. So erhielten wir die Formel $\operatorname{ch} b=$ $\operatorname{sh} d \operatorname{sh} e$.

Jetzt wollen wir auf eine Formel der zweiten Klasse, etwa $\operatorname{ch} c=\operatorname{cth} d \operatorname{cth} b$ zusteuern. Wir müssen aus den noch immer gültigen Formeln (2) neben $a$ jetzt auch noch $e$ eliminieren. Dabei gehen wir genauso vor wie in 2.2.4. Anstelle von $\operatorname{sh}^{2} e$ in (3) schreiben wir $\mathrm{ch}^{2} e-1$ und lösen dann die sich ergebenden Gleichungen nach $\operatorname{ch}^{2} e$ auf. Wir begnügen uns damit, die Gleichungen $\mathrm{I}^{*}$ und $\mathrm{V}^{*}$ auf diese Weise zu behandeln.

$$
\mathrm{I}^{* *} \quad \operatorname{ch}^{2} e=1+\frac{\operatorname{ch}^{2} c \operatorname{sh}^{2} b}{\operatorname{ch}^{2} d}
$$




$$
\mathrm{V}^{* *} \quad \operatorname{ch}^{2} e=1+\frac{\operatorname{ch}^{4} b \operatorname{sh}^{2} c}{\operatorname{ch}^{2} b+\operatorname{ch}^{2} c \operatorname{sh}^{2} d} .
$$

Durch Gleichsetzen von $\mathrm{I}^{* *}$ und $\mathrm{V}^{* *}$ wird nun $e$ eliminiert.

$$
\begin{gathered}
1+\frac{\operatorname{ch}^{2} c \operatorname{sh}^{2} b}{\operatorname{ch}^{2} d}=1+\frac{\operatorname{ch}^{4} b \operatorname{sh}^{2} c}{\operatorname{ch}^{2} b+\operatorname{ch}^{2} c \operatorname{sh}^{2} d} \\
\Longrightarrow \quad \operatorname{ch}^{4} b \operatorname{sh}^{2} c \operatorname{ch}^{2} d=\operatorname{ch}^{4} c \operatorname{sh}^{2} b \operatorname{sh}^{2} d+\operatorname{ch}^{2} b \operatorname{ch}^{2} c \operatorname{sh}^{2} b .
\end{gathered}
$$

Division mit $\operatorname{sh}^{2} d \operatorname{sh}^{2} b$ liefert

$$
\operatorname{cth}^{2} b \operatorname{cth}^{2} d=\frac{\operatorname{ch}^{2} c}{\operatorname{ch}^{2} b \operatorname{sh}^{2} c}\left(\operatorname{ch}^{2} c+\frac{\operatorname{ch}^{2} b}{\operatorname{sh}^{2} d}\right) .
$$

Jetzt greifen wir zur weiteren Umformung auf zwei bereits bekannte Formeln zurück.

Die zweite Formel 1. Klasse in (1): $\operatorname{sh}^{2} e=\frac{\operatorname{ch}^{2} b}{\operatorname{sh}^{2} d}$.

Die Formel IV in (2): $\operatorname{ch}^{2} b \operatorname{sh}^{2} c=\operatorname{ch}^{2} a \operatorname{sh}^{2} e$.

Wir setzen ein und erhalten

$$
\operatorname{cth}^{2} b \operatorname{cth}^{2} d=\operatorname{ch}^{2} c\left(\frac{\operatorname{ch}^{2} c}{\operatorname{ch}^{2} a \operatorname{sh}^{2} e}+\frac{1}{\operatorname{ch}^{2} a}\right) .
$$

Und nochmals verwenden wir eine bekannte Formel.

Die dritte Formel 1. Klasse in (1): $\operatorname{ch}^{2} c=\operatorname{sh}^{2} a \operatorname{sh}^{2} e$. Damit erhalten wir

$$
\operatorname{cth}^{2} b \operatorname{cth}^{2} d=\frac{\operatorname{ch}^{2} c}{\operatorname{ch}^{2} a}\left(\operatorname{sh}^{2} a+1\right)=\operatorname{ch}^{2} c .
$$

Weil die zum Beweis verwendeten Formeln aus (1) und (2) alle gelten, so tun dies auch alle Formeln der Klasse 2 aus (1). Eine Behandlung kritischer Fälle ist nicht erforderlich.

Die für beide Formelklassen gültigen Aussagen (b) und (c) aus 2.2 lassen sich zu einem nicht-trivialen Lemma zusammenfassen.

\subsection{Lemma}

Drei gleichlange Seiten in einem 5-Rechteck induzieren fünf gleich lange Seiten, also ein reguläres 5-Rechteck. 


\subsection{Korollar}

Gibt man zwei Stücke eines 5-Rechtecks vor, so sind durch die Gleichungen (2) alle überigen Stücke bestimmt. Damit läßt sich ein Korollar formulieren.

Es gibt unendlich viele $\left(\infty^{2}\right)$ nicht $H$-kongruente 5-Rechtecke.

Die regulären 5-Rechtecke sind alle zu einander H-kongruent.

\section{Was gibt es für den Leser noch zu Tun?}

Beweisen Sie durch Konstruktion im Klein-Modell die Existenz von 6-Rechtecken! Wie lang ist eine Seite im regulären 6-Rechteck? Suchen Sie bei 6-Rechtecken nach Formeln die denen von Sommerville ähnlich sind und beweisen Sie diese! Existieren in der hyperbolischen Geometrie $n$-Rechtecke mit $n>6$ ?

Und noch etwas für hyperbolische Fliesenleger: Läßt sich die hyperbolische Ebene mit $n$-Rechtecken parkettieren?

\section{Literatur}

[1] G. Buchmann, Nichteuklidische Elementargeometrie, Stuttgart, 1975.

[2] R. Baldus and F. Lobell, Nichteuklidische Geometrie, Berlin, 1953.

[3] D. M. Y. Sommerville, The elements of Non-Euclidean Geometry, New York, 1958.

[4] H. Zeitler, Hyperbolische Geometrie, München, 1970.

H. ZEITLER

BAYREUTH

GERMANY

(Received July 16, 2002) 